\title{
Editorial
}

\section{ANESTHESIOLOGY: A Spurt of Growth}

The last four decades have witnessed a remarkable growth in anesthesiology as a specialty in India. The changes have been varied and remarkable.

A simple combination of a basic anesthesia machine and an electrocardiogram (ECG) monitor has now evolved into an anesthesia workstation with all possible parameters monitored. Intensive monitoring definitely allows us to achieve greater safety assuming proper vigilance.

All major hospitals now have a dedicated preoperative anesthesia checkup unit that ensures

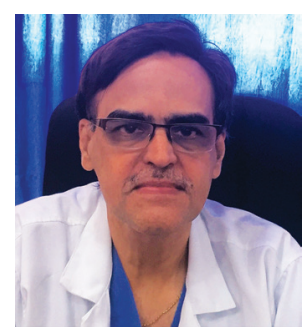
adequate optimization via recognition and treatment of comorbidities. Optimization translates to a lesser risk of complications. The anesthesia provider is also protected as litigation in the post-CPA (Consumer Protection Act) era is a distinct possibility and insurance companies consider anesthesia as a high risk specialty.

The anesthetist is now increasingly involved in the designing and management of operating suites. It is generally accepted that he/she is the ideal person to maintain a high level of efficiency and productivity.

Our specialty has stepped out of the confines (and safety) of the operating room to cater to radiology and endoscopy suites besides cardiac catheterization laboratory. Various factors compromise safety in these locations and the anesthetist has to be extra vigilant to ensure high standards of care.

Critical care has long been a part of anesthesia curricula. Medical Council of India (MCI) has recognized critical care medicine as a separate subject since 2010. Although critical care units require multidisciplinary care, it is not uncommon to find such units headed by an anesthetist.

Pain management as a specialty is in it's nascent stages in our country. The introduction of special courses in pain management will lead to rapid expansion and due recognition.

Subspecialities like cardiac anesthesia, neuroanesthesia and pediatric anesthesia have come of age with DM courses being offered by major institutes. Research and publications have assumed high priority. The last few years have seen candidates with higher ranks in common entrance tests opting for anesthesia. This trend will undoubtedly continue till anesthesiology is one of the top choices.

SK Gvalani

Editor-in-Chief

Research and Innovation in Anesthesia Journal Head, Department of Anesthesia

RN Cooper Hospital

Mumbai, Maharashtra, India 